\title{
LOS APRENDIZAJES DE COMPETENCIAS GENÉRICAS DE LOS FUTUROS GRADUADOS EN GEOGRAFÍA: UNA REFLEXIÓN INICIAL Y PAUTAS PARA SU DISEÑO Y ENSEÑANZA*
}

\author{
Ana Isabel Escalona Orcao y Blanca Loscertales Palomar \\ Departamento de Geografía y Ordenación del Territorio, Facultad de Filosofía y Letras, \\ Universidad de Zaragoza. C/ Pedro Cerbuna, 12, 50009-Zaragoza \\ aescalon@unizar.es_bloscer@unizar.es
}

\begin{abstract}
Resumen: Este artículo comienza con diversas consideraciones sobre las competencias genéricas y su importancia en el título de grado de Geografía y Ordenación del Territorio, ante la próxima entrada en vigor del Espacio Europeo de Educación Superior. Su objetivo se centra en presentar un modelo metodológico orientado a ayudar a los profesores que deberán remodelar sus enseñanzas y rediseñar las asignaturas que van a impartir en un corto espacio de tiempo, al tener que introducir en sus programas cuestiones para el aprendizaje de competencias genéricas. Sus autoras presentan diferentes ejemplos sobre el modo de establecer los resultados de aprendizaje, de enfocar la evaluación o de seleccionar las actividades académicas que pueden proponerse a los estudiantes para facilitar la consecución de los resultados previstos. Por último, presta también atención al modo de secuenciar estas cuestiones para cada uno de los niveles de aprendizaje o de insertarlas en las asignaturas de Geografía.
\end{abstract}

Palabras clave: Título de grado en Geografía, Competencias genéricas, Metodología para el aprendizaje, Espacio Europeo de Educación Superior.

\begin{abstract}
Teaching, learning and assessment of key skills (competences) is an important step in the process of designing new degrees for the European Higuer Education Area. The aim of this article is to provide staff teaching Geography with a methodological model for including skills-based academic activities within the Geography curriculum and modules. Examples are given of statement of learning outcomes, developing of assesement methods, writing of assessment criteria and choice of teaching activities. We add practice material to help staff address the several issues involved in developing the skills of their students. We finish emphasising skills learning and inviting teachers to undertake this task
\end{abstract}

Key words: skills, generic competences, learning methodologies, European Higuer Education Area.

\footnotetext{
* Recibido: 14-10-05. Aceptado: 10-1-06.
} 


\section{Introducción}

En el marco del proceso europeo de convergencia universitaria, el aprendizaje de competencias es un referente común para la formación de los estudiantes, que, poco a poco, se va incorporando a la práctica docente. El principal objetivo de este artículo es proporcionar a los profesores que deberán impartir el Título de Grado en Geografía, una base metodológica adecuada y susceptible de ser aplicada en el diseño de la enseñanza universitaria y de actividades para el aprendizaje de competencias genéricas en sus respectivas asignaturas. Lo que se expone a continuación es parte de los resultados de la investigación llevada a cabo por las autoras en el marco de dos proyectos de innovación docente sobre competencias genéricas, que fueron seleccionados por el Instituto de Ciencias de la Educación de la Universidad de Zaragoza en las convocatorias de 2003 y de $2004 .{ }^{1}$ Los proyectos se centraban en dos competencias genéricas, ${ }^{2}$ trabajo en equipo y comunicación, cuya importancia ha quedado repetidamente reflejada en estudios recientes sobre la adecuación de la formación que reciben los universitarios a las demandas laborales (Fundación Universidad-Empresa, 1999; Fundación BBVA, 2004).

Los resultados obtenidos en nuestro primer proyecto sobre el trabajo en equipo han quedado suficientemente expuestos en el libro Actividades para la enseñanza y aprendizaje de competencias genéricas en el marco del Espacio Europeo de Educación Superior (Escalona y Loscertales, 2005). En este artículo, además de una reflexión inicial, como afirma el título, se aportan algunos de los materiales inéditos elaborados en ambos proyectos, para que el profesor interesado en estas cuiestiones disponga de una herramienta útil que pueda utilizar en su tarea educativa diaria. El artículo comienza situando el aprendizaje de competencias en el diseño de las nuevas titulaciones, dejando claramente patente su importancia como elemento clave de las mismas y la oportunidad de su incorporación a las enseñanzas universitarias. A continuación se destaca la importancia de las competencias genéricas en el título de grado de Geografia y Ordenación del Territorio. La parte central del texto se refiere al reto de incluir el aprendizaje de las competencias genéricas en el aula, e incorporarlo en las respectivas disciplinas. Para ello, se incluyen diferentes ejemplos sobre cómo establecer los resultados de aprendizaje, cómo enfocar su evaluación, qué actividades

1 Las autoras agradecen al Instituto de Ciencias de la Fducación de la Universidad de Zaragoza, patrocinador de los proyectos, y a la dirección del Departamento de Geografía y Ordenación del Territorio la ayuda dispensada para la realización de la experiencia de innovación docente, cuyos frutos quechan parcialmente expuestos en está publicación.

2 En la convocatoria de proyectos de Innovación Docente realizada por el Instituto de Ciencias de la Fducación de la Universiclad de Zaragoza en el ano 2003 fue seleccionado el proyecto "Discnos y Secuenciación de Actividades Académicas Dirigadas para el aprendizaje de Competencias genéricas en Geografía", cuyo desarrollo posterior se centró en la competencia lrabajo en equipo. A la convocatoria de 2004 se presentó otro proyecto titulado "Diseno y realización de actividades académicas para la enseñanzal y aprendizaje de competencias de comunicación", que también fue seleccionado. 
académicas se pueden proponer a los estudiantes para facilitar la consecución de los resultados señalados, cómo secuenciarlas para cada nivel o cómo insertarlas en las asignaturas de Geografía. Deliberadamente se han omitido las cuestiones conceptuales y teóricas, ya que el interesado puede recurrir a la bibliografía existente para profundizar en dichos aspectos (Fallows y Steven, 2000; García Valcárcel, 2001; Monereo y Pozo, 2003; Zabalza, 2003; VV.AA., 2005). El artículo termina con unas consideraciones finales sobre los aspectos analizados y lo que va a suponer la inserción de las universidades en el Espacio Europeo de Educación Superior.

\section{Las competencias genéricas y su importancia en el título de grado de Geografía y Ordenación del Territorio}

\subsection{La competencias genéricas en el diseño de los nuevos títulos de grado}

El proceso de convergencia en el que se encuentran inmersas las universidades europeas tiene su origen, como es sabido, en la aspiración política de crear en Europa un área de educación superior integrada, paralela a las ya existentes o en curso en los ámbitos económico y social. El proceso encuentra un decisivo impulso con el proyecto Tuning Educational Structures in Europe, conocido como Tuning, cuyo objetivo básico es sintonizar las estructuras y los programas de la enseñanza universitaria ante la perspectiva de la próxima entrada en vigor del espacio educativo europeo común y facilitar la comparación y homologación de los estudios. Otros factores que han contribuido a poner en marcha ese proceso son la movilidad de los titulados y su necesidad de que la educación recibida por todos sea compatible, comparable y competitiva, así como las exigencias de los empresarios y empleadores sobre el significado de un título emitido por una universidad y la capacitación concreta que da a quien lo ha conseguido (VV.AA, 2003). Un elemento fundamental de la Convergencia Universitaria es el diseño de los nuevos títulos de grado de manera que

Tabla 1. Etapas básicas del diseño de los títulos de grado. Fuente: Wagenaar, 2003.

1. Identificación de las necesidades sociales relacionadas con la titulación.

2. Definición de los perfiles académicos y profesionales.

3. Establecimiento de resultados de aprendizaje: competencias.

4. Traslado a los curriculos: inserción en los contenidos de la titulación y estructuración de los mismos (cursos, módulos, créditos).

5. Selección de las metodologías para su enseñanza, aprendizaje y evaluación.

6. Establecimiento de programas para asegurar la calidad de todo el proceso. 
faciliten la consecución de los objetivos previstos. Para ello se ha recomendado que todas las titulaciones se elaboren siguiendo una metodología común que consta de las etapas indicadas en la tabla 1.

Como se deduce de la tabla adjunta, los contenidos y estructura de la titulación así diseñada (Etapa 4) aparecen supeditados a la determinación previa de las necesidades sociales que viene a satisfacer dicha titulación (Etapa 1); al establecimiento de los rasgos que caracterizan a sus egresados -perfiles- (Etapa 2); y al enunciado de qué resultados o conjuntos de competencias -conocimientos, capacidades y habilidades- debe proporcionar el aprendizaje para que los titulados afronten con una auténtica preparación las tareas propias de su perfil profesional (Etapa 3). El concepto de competencia adquiere así todo su significado ya que expresa lo que el estudiante ha de dominar, comprender y demostrar al término del grado, o de cada uno de los cursos que lo componen. Por otra parte, permite seleccionar entre una amplia gama de posibilidades, los conocimientos apropiados para determinados fines. Por último, las competencias facilitan la comprensión del currículo y el establecimiento de puntos comunes de referencia respecto del nivel de formación alcanzado por el titulado sea cual fuere el lugar donde ha cursado los estudios (VV.AA., 2003)

El titulado deberá de haber adquirido dos clases de competencias: las llamadas específicas -o propias de su campo de estudio - y las genéricas -comunes para cualquier titulación, llamadas también, por ello, transversales (VV.AA, 2003). La incorporación efectiva a los aprendizajes de estas últimas ha podido extrañar al profesorado universitario por referirse a cuestiones no habituales en su quehacer ordinario, tales como potenciar la capacidad de comunicación de los estudiantes, o ayudarles a cooperar de forma efectiva, o a generar nuevas ideas. Pero todos somos conscientes de su importancia para facilitar el empleo y para formar ciudadanos en el pleno significado del término, capaces de actuar como personas y de afrontar sus responsabilidades sociales. Por ello, la Universidad debe superar el enfoque simplista de la formación para la cualificación profesional y preparar a los futuros profesionales prestando atención también a la multiplicidad de saberes, de cultura, virtudes y valores que la sociedad necesita (Cruz, 2005).

\subsection{Las competencias genéricas en el proyecto de título de grado en Geografía y Ordenación del Territorio}

¿Qué competencias genéricas deberán aprender en su día los estudiantes del grado en Geografía? ¿Cuáles pueden ir ya incorporando los profesores en sus respectivas asignaturas? La respuesta a ambas preguntas es todavía incierta porque en la mayoría de las Universidades, aún no se ha iniciado el diseño de los títulos de grado. No obstante se dispone ya de elementos suficientes como para que los profesores 
interesados puedan comenzar a planificar esta parte de su tarea. Una referencia básica es la relación de treinta competencias genéricas aportada en el Informe final de la fase primera del proyecto Tuning clasificadas en las siguiente categorías (VV.AA, 2003):

- Competencias instrumentales: Son aquellas que tienen una función instrumental, y que pueden ser a su vez cognoscitivas, metodológicas, tecnológicas y lingüísticas.

- Competencias interpersonales: Aquellas que tienden a favorecer los procesos de interacción social y comunicación.

- Competencias sistémicas: o combinación de comprensión, sensibilidad y conocimiento, para permitir al individuo ver cómo las partes de un todo se relacionan y se agrupan.

Otra referencia importante, es el proyecto de título de grado en Geografía y Ordenación del Territorio, elaborado por un amplio grupo de especialistas en esta disciplina de diversas universidades y publicado como Libro Blanco de Geografía y Ordenación del Territorio (Agencia Nacional de Evaluación y Acreditación, 2004). ${ }^{3}$ En él se contienen ya algunas propuestas relativas a las etapas iniciales del proceso descrito en la tabla 1. Cabe citar, por ejemplo, los perfiles o ámbitos de inserción laboral de los futuros egresados: Investigación, educación y divulgación geográficas; Tecnologías de la información geográfica; Medio físico, recursos naturales y medio ambiente; Planificación y gestión territorial; Análisis territorial de la población y demografía; Desarrollo regional y local. En lo referente a las competencias genéricas, el equipo que elaboró el proyecto consideró oportuno añadir a las sugeridas para el conjunto europeo algunas más, siempre en relación con habilidades y actitudes propias de los perfiles profesionales propuestos. La tabla 2 recoge las diferentes competencias genéricas incluidas en el Libro Blanco.

\subsection{Importancia formativa de las competencias genéricas para los futuros titulados}

El diseño del aprendizaje de competencias genéricas requiere determinar cuales pueden seleccionarse en el marco de una titulación. Lógicamente no todas las com-

3 El equipo redactor del Libro estaba coordinado por el Dr. Tulla de la Universidad de Barcelona, y de él formaban parte profesores de Geografía de las universidades de Valencia, Sevilla, Autónoma y Complutense de Madrid, Cantabria y las dos barcelonesas: Autónoma y Central. También se integraron en el equipo redactor sendos representantes de la Asociación de Geógrafos Españoles y del Colegio de Geógrafos. 
Tabla 2. Relación de competencia genéricas contempladas en el proyecto del título de grado en Geografía y Ordenación del Territorio.

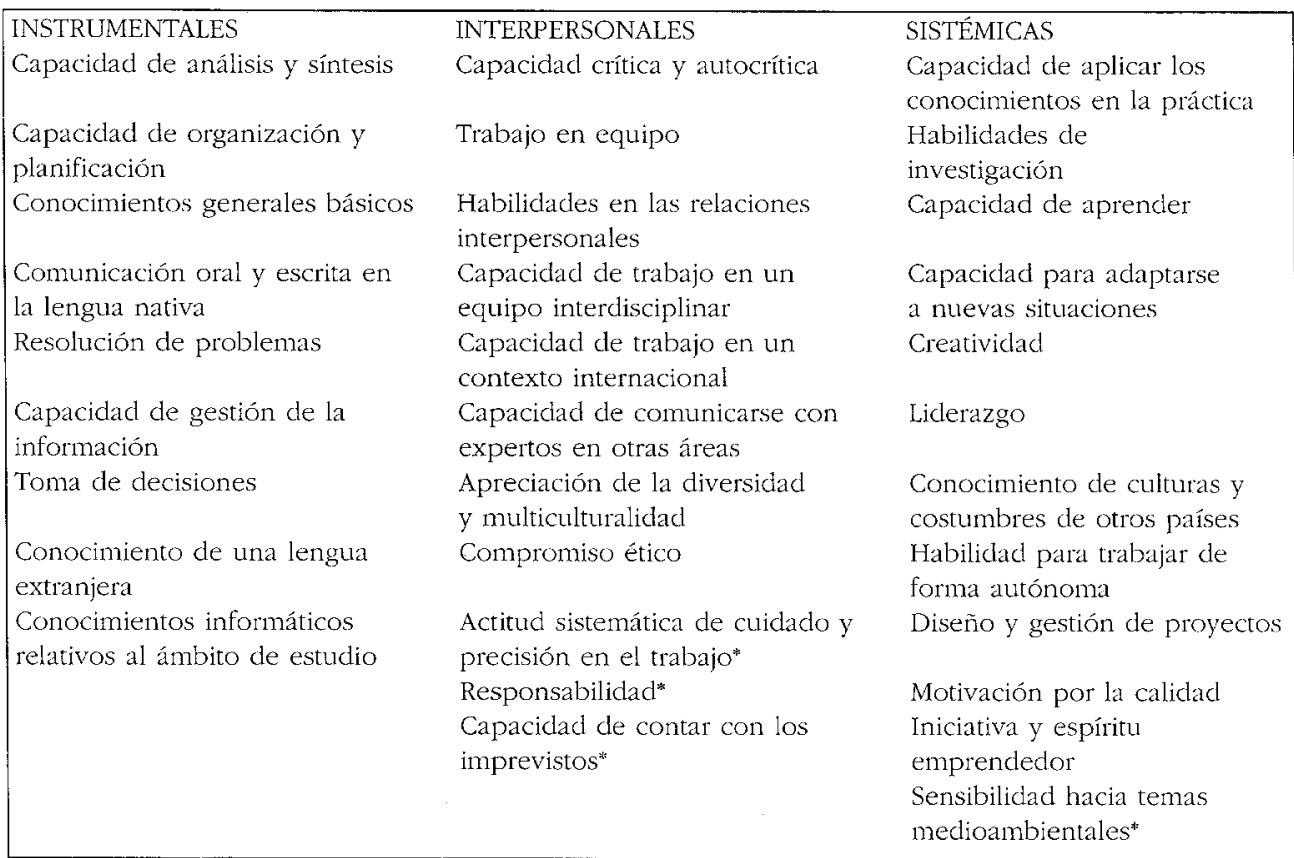

* Competencia añadida por el equipo de especialistas que elaboró el proyecto del título de grado en Geografía y Ordenación del Territorio. Fuente: VV.A, 2003: 83 y 84; Agencia..., 2004. Elaboración propia.

petencias genéricas tiene la misma importancia para el futuro desarrollo de la actividad profesional y, por tanto, deberá de ser diferente el esfuerzo formativo requerido para cada una de las competencias elegidas. Su diferente interés quedó ya patente en el informe Tuning, en el que se publicaron los resultados de una encuesta de valoración de las competencias genéricas dirigida a empresarios, graduados y académicos. Los encuestados generalmente solían coincidir en que las competencias genéricas más importantes para su trabajo son: capacidad de análisis y síntesis; capacidad de aprender; capacidad de resolver problemas; capacidad para aplicar los conocimientos en la práctica; capacidad para adaptarse a nuevas situaciones; preocupación por la calidad; habilidades de gestión de la información capacidad para trabajar de forma autónoma; trabajo en equipo (VV.AA., 2003). En algunas respuestas los académicos diferían de los dos grupos restantes de profesionales seleccionados citados. Por ejemplo, los académicos puntuaron en primer lugar los conocimientos generales básicos; su valoración de las destrezas informáticas básicas y de las competencias interpersonales, fue, sin embargo, inferior a la de los dos. grupos restantes. 
Bastante semejantes a los anteriores son los resultados de la encuesta realizada por el grupo de especialistas españoles que elaboró el proyecto de título de grado en Geografía y Ordenación del Territorio (Agencia..., 2004). Según la muestra de licenciados, profesionales de la geografia - universitarios y no universitarios - y empleadores de geógrafos que fueron consultados, las competencias genéricas consideradas más necesarias para desarrollar las tareas propias del geógrafo son: capacidad de análisis y síntesis (puntuada con 3'6 sobre 4); actitud sistemática de cuiclado y precisión en el trabajo, responsabilidad (ambas con 3'5); capacidad de organización y planificación; capacidad crítica y autocrítica (ambas con 3'4); comunicación oral y escrita; sensibilidad medioambiental, motivación por la calidad, habilidad para trabajar de forma autónoma (todas puntuadas con 3'3); resolución de problemas, trabajo en equipo, capacidad de gestión de la información, aprendizaje autónomo, capacidad de adaptación a nuevas situaciones (puntuadas todas con 3'2).

De los resultados de una encuesta realizada a los estudiantes participantes en el primero de los proyectos de innovación docente ya mencionado, se deduce que sus respuestas, se asemejan bastante a las anteriores. El proyecto, coordinado por las

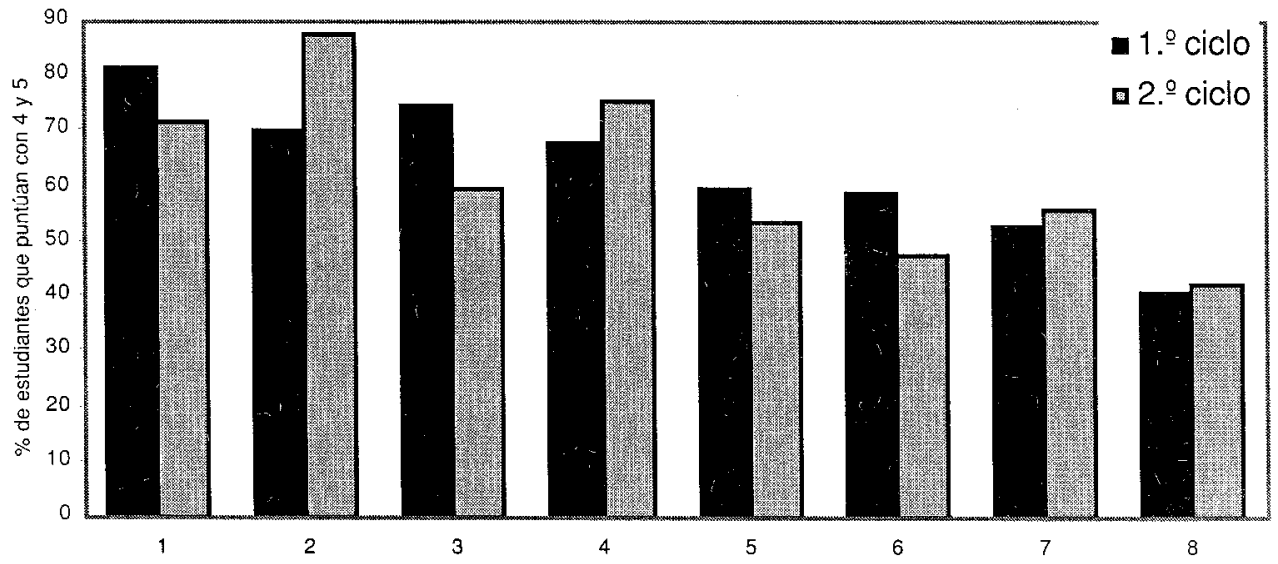

Figura 1. Capacidades y competencias según su importancia para la formación. Fuente: Elaboración propia a partir de la encuesta a los estudiantes.

\footnotetext{
1. Seleccionar la clocumentación y materiales adecuados para preparar un argumento.

2. Utilizar con facilidad las nuevas tecnologias para presentar una información.

3. Comunicar con eficacia de forma oral y escrita diversas cuestiones.

4. Saber trabajar en equipo.

5. Aprender por sí mismo.

6. Reflexionar criticanente sobre su proceso de aprendiziaje

7. Interpretar información cle carácter numérico y estadístico.

8. Aplicar las herramientas básicas para un análisis cuantitativo de la información.
} 
autoras, fue llevado a cabo en el Departamento de Geografía de la Universidad de Zaragoza. ${ }^{4}$ En sus respuestas, quedaba patente una alta valoración de determinadas competencias y capacidades genéricas (figura 1). Especialmente destacaban las relacionadas con el tratamiento de la información geográfica, y la capacidad de comunicación. Les interesaba también ser formados para saber trabajar en un equipo y para estudiar o aprender autónomamente. Esta opinión era confirmada por más de un $70 \%$ de los estudiantes encuestados (Escalona y Loscertales, 2005).

La valoración desciende en el caso de las restantes competencias y capacidades, ya que el porcentaje de estudiantes encuestados que las puntúan con el nivel 4 y 5 , es decir, el más elevado, es siempre inferior al 60\%. En los dos primeros casos se trata de cuestiones relacionadas con su propio proceso de aprendizaje y con la reflexión crítica sobre el mismo. Las situadas en los dos últimos lugares hacen referencia a la interpretación y utilización de información numérica y a la capacidad de aplicación de herramientas matemáticas básicas a la información geográfica. ${ }^{5}$

\subsection{Evidencias del déficit formativo en competencias genéricas y oportunidad de su incorporación plena a los contenidos del título de grado en Geografía.}

La importancia concedida a las competencias genéricas por los diversos estamentos universitarios y no universitarios tanto de España como del resto de Europa, contrasta con la percepción de que la formación recibida en las universidades sobre tales cuestiones es insuficiente, por lo que debe ser reforzada cuando se diseñen las nuevos títulos. La tabla 3 se basa de nuevo en la encuesta realizada para la elaboración del proyecto de título de grado en Geografía y expresa en forma de déficit la diferencia entre la importancia formativa de las competencias más valoradas y la enseñanza recibida por los graduados y académicos españoles consultados. Queda clara la afirmación anterior de que las enseñanzas han sido generalmente inferiores a las necesidades formativas de los distintos perfiles profesionales. Esta opinión es compartida por todos los profesionales seleccionados en la muestra para la aplicación de

4 La encuesta fue repartida a los estudiantes matriculados en la asignaturas, de primer y segundo ciclo, impartidas por los profesores mienbros del equipo y como resultado de la misma se obtuvieron 76 cuestionarios.

5 En ambos casos, curiosamente, los estudiantes muestran un menor interés por su formación en estas capacidades, cada vez más necesarias, dada la complejidad de la información estadística existente. Sin embargo, ellos no lo perciben así, mostrando un cierto desconocimiento del perfil profesional que, en el desempeño de muchos trabajos, les va a ser requerido. Llama también la atención la menor percepción de la necesidad de formación en tales competencias entre los estudiantes de segundo ciclo. Pensamos que este hecho es preocupante, ya que denota una menor apreciación de aspectos que les ayudarían a perfeccionar su proceso de aprendizaje (v. 5 y 6 ) o a mejorar sus comunicación y su capacidad para saber presentar el material (v. 1, y 3.) (Escalona y Loscertales, 2005). 
Tabla 3. Déficit formativo en las principales competencias genéricas.

\begin{tabular}{|c|c|c|c|c|}
\hline \multirow[t]{2}{*}{ Competencias } & \multicolumn{4}{|c|}{ Déficit formativo* } \\
\hline & Nulo o bajo & Medio & Alto & Muy alto \\
\hline Instrumentales & - & $\begin{array}{l}\text { Capacidad de } \\
\text { análisis y síntesis }\end{array}$ & $\begin{array}{l}\text { Comunicación } \\
\text { oral y escrita en } \\
\text { lengua nativa } \\
\text { Capacidad de } \\
\text { organización y } \\
\text { planificación } \\
\text { Resolución de } \\
\text { problemas } \\
\text { Capacidad de } \\
\text { gestión de la } \\
\text { información }\end{array}$ & $\begin{array}{l}\text { Conocimientos } \\
\text { informáticos } \\
\text { básicos }\end{array}$ \\
\hline Interpersonales & - & $\begin{array}{l}\text { Trabajo en equipo } \\
\text { Compromiso ético } \\
\text { Capacidad crítica y } \\
\text { autocrítica }\end{array}$ & & $\begin{array}{l}\text { Trabajo en equipo } \\
\text { de carácter } \\
\text { interdisciplinar }\end{array}$ \\
\hline Sistémicas & $\begin{array}{l}\text { Sensibilidad } \\
\text { hacia temas } \\
\text { medioambien- } \\
\text { tales* }\end{array}$ & $\begin{array}{l}\text { Aprendizaje } \\
\text { autónomo }\end{array}$ & $\begin{array}{l}\text { Capacidad para } \\
\text { trabajar } \\
\text { autónomamente }\end{array}$ & $\begin{array}{l}\text { Adaptación a } \\
\text { nuevas situaciones }\end{array}$ \\
\hline & & $\begin{array}{l}\text { Habilidades de } \\
\text { investigación }\end{array}$ & $\begin{array}{l}\text { Creatividad } \\
\text { Motivación por } \\
\text { la calidad } \\
\text { Actitud sistemática } \\
\text { de cuidado y } \\
\text { precisión en el } \\
\text { trabajo* } \\
\text { Responsabilidad } \\
\text { Capaciad de aplic } \\
\text { conocimientos a } 1 \\
\text { práctica } \\
\text { Capacidad de } \\
\text { comunicarse de } \\
\text { manera efectiva } \\
\text { con no expertos }\end{array}$ & $\begin{array}{l}\text { Capacidad de } \\
\text { contar con los } \\
\text { imprevistos } \\
\text { Diseño y gestión } \\
\text { de proyectos } \\
\text { a } \\
\text { ar }\end{array}$ \\
\hline
\end{tabular}

* Diferencia en una competencia dada entre el nivel de formación recibido (puntuado según la siguiente cscala: 1. ningún nivel de aprendizaje; 2, nivel básico; 3, nivel suficiente; 4, nivel completo) y el considerado necesario para el desempeño profesional (puntuado según la siguiente escala: 1, competencia que no es necesaria para desarrollar tareas profesionales y que no requiere ningún nivel de aprendizaje; 2 , competencia para la que se requiere un nivel básico de aprendizaje; 3 , competencia que requiere un nivel suficiente; 4 , competencia que requiere un nivel completo de aprendizaje). Pautas de interpretación: Déficit nulo o bajo: <-04 o valores positivos; medio: -0 '5<céficit<-0 75 ; alto: $-075<$ cléficil<-1; muy alto: déficit>-1'1 (Fuente: Agencia..., 2004. Elaboración propia). 
la encuesta, si bien la apreciación de los graduados de menos de cinco años es más optimista, lo que indica que ya contaron con mejor formación en esta cuestiones que sus predecesores. ${ }^{6}$

Los actuales estudiantes de Geografía necesitan ser formados en estas cuestiones. En la figura 2, elaborada a partir de la encuesta realizada por las autoras de este artículo (v. supra), puede observarse que la proporción de estudiantes que perciben tener en grado alto o muy alto las competencias sobre las que se les consulta, es baja. Sólo en cinco de las dieciséis capacidades de la lista, el 50\% o más de los alumnos afirma que su nivel es alto o muy alto; es el caso de Sociabilidad (79,5\%), Leer $(75,5 \%)$ Escuchar (61\%), Responsabilidad (55\%) y Autonomía (54,7\%). Hay un segundo grupo de capacidades en las que la proporción de estudiantes que afirman poseerlas en alto grado alcanza valores siempre inferiores al $50 \%$ y superiores al $30 \%$. Son las aptitudes Escribir (42,5\%), Hablar (40\%), Estima personal (40\%), Pensar de forma creativa (38\%), Tomar decisiones (37\%), Resolver problemas (37\%) y Razonamiento (33\%). Puede apreciarse un tercer grupo de aptitudes en las que el porcentaje de los estu-

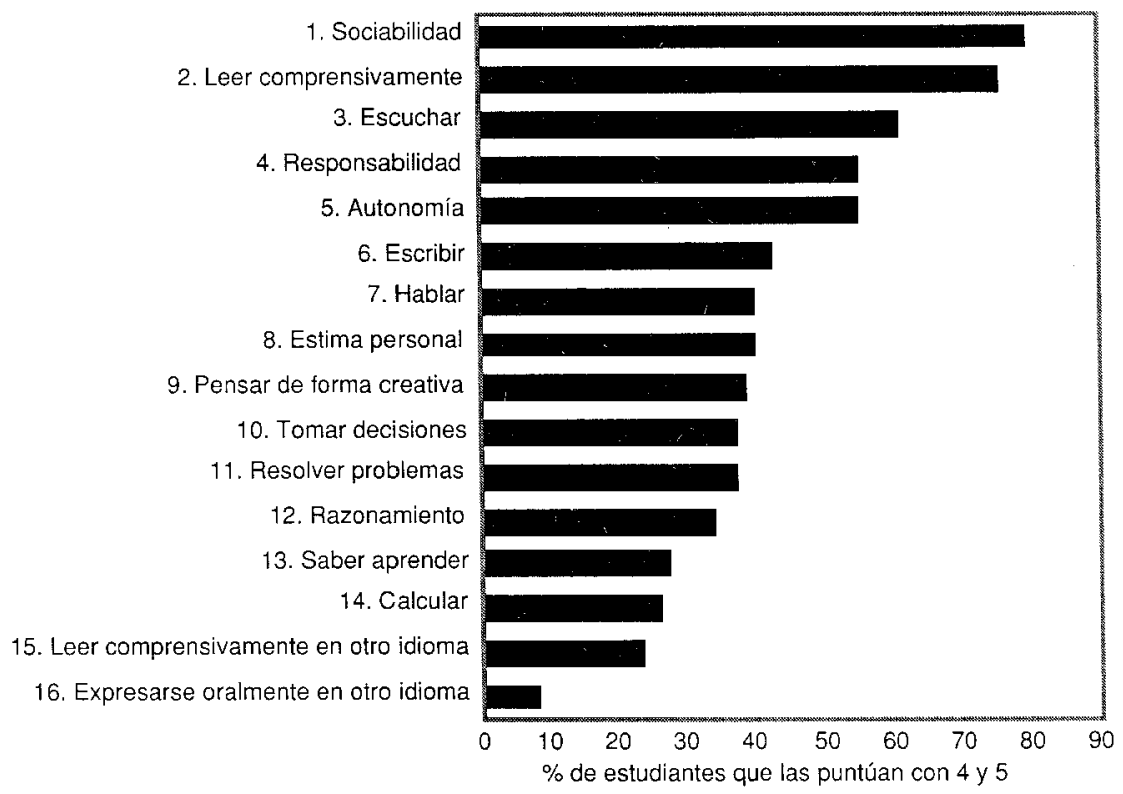

Figura 4. Percepción de las aptitudes personales básicas. Fuente: Elaboración propia a partir de la encuesta a los estudiantes.

6 Quienes deseen más información sobre la realización la metodología y resultados detallados de la citada encuesta en relación con las competencias genéricas, la encontrarán en los capítulos 8 y 11 cle la publicación del proyecto del título de grado (Agencia..., 2004). 
diantes que expresa tener un nivel alto o muy alto es inferior al 30\%; son Saber aprender (27\%), Calcular (25,9\%), Leer comprensivamente en otro idioma (23\%) y Expresarte en otro idioma (7,9\%) (Escalona y Loscertales, 2005).

Como conclusión cabe decir que los resultados expuestos justifican la inserción de competencias genéricas de forma explícita en los contenidos de la titulación, no sólo para potenciar las diferentes destrezas propias de la profesión sino también para trabajar en todas el conjunto de habilidades de pensamiento recogidas por Bloom en su taxonomía, tanto las más elementales -conocimiento, comprensión y aplicación -como las complejas -análisis, síntesis y evaluación (Barton, 1996). Sólo así los futuros titulados en Geografía podrán abordar con éxito su propio proceso de formación y su posterior inserción profesional. Con los epígrafes que siguen queremos facilitar a los profesores universitarios el desarrollo de actividades académicas encaminadas a tales fines.

\section{Pautas para la enseñanza y el aprendizaje de competencias genéricas en las asignaturas de Geografía}

\subsection{Consideraciones previas}

Las consideraciones llevadas a cabo en esta parte del artículo se refieren a una fase muy avanzada del proceso de diseño de las titulaciones descrito en la tabla 1, cuando los profesores tengan que incorporar el aprendizaje de las competencias genéricas en los programas de las asignaturas que les corresponda impartir. Con anterioridad, las autoridades académicas universitarias responsables del diseño de la titulación deberán haber dado ya una solución adecuada a cuestiones importantes sobre la estructura de las enseñanzas; es decir, sobre su organización en cursos, módulos y créditos. La tabla 4 hace referencia a algunas de ellas.

Son cuestiones de gran interés y que sin duda van a condicionar el futuro trabajo de los profesores una vez que entren en vigor las nuevas titulaciones. Quienes desarrollen su tarea docente en la titulación de Geografía tendrán que esperar a que las respectivas universidades elaboren los títulos de grado en Geografía y Ordenación del Territorio para ver cómo se resuelven algunas de las incógnitas. ${ }^{7}$ Sólo entonces podrá

7 En el Libro Blanco del proyecto de la titulación (Agencia..., 2004) hay una propuesta de asignación del aprendizaje de determinadas competencias genéricas a los grupos de contenidos comunes obligatorios para los que se consideran más necesarias. Es una propuesta que debe tomarse con cautela ya que se basa en una discutible apreciación de tales necesidades por parte de quienes fueron encuestados para ello (v. capítulos 11). 
Tabla 4. Cuestiones de reflexión en la incorporación de las competencias genéricas a los nuevos títulos de grado. Fuente: Elaboración propia a partir de Livingstone y Matthews, 2000; y V.AA, 2003.

1. ¿Qué habilidades y capacidades desearíamos que los estudiantes hubieran adquirido al final de sus estudios?

2. ¿Cuántas pueden desarrollarse en el programa de una titulación?

3. ¿Van a seleccionarse tales capacidades según titulaciones o van éstas a asumir las que establezcan en sus idearios las universidades respectivas?

4. ¿Cómo se va a insertar su aprendizaje en el currículo de la titulación?

5. ¿Qué resultados de aprendizaje específicos van a establecerse en cada uno de los años de formación?

6. ¿Qué evidencias de progreso se van a establecer en cada nivel y cómo van a evaluarsé?

7. ¿Van a ser objeto de asignaturas o módulos específicos o se va a integrar su aprendizaje en las ya existentes?

procederse a diseñar una metodología concreta para la enseñanza, aprendizaje y evaluación de las competencias genéricas en las diferentes asignaturas que impartamos. Esta fase es la más importante de todo el proceso, y conviene que los profesores - sus principales agentes- estén preparados.

\subsection{Los diferentes contextos del aprendizaje de competencias}

Las pautas que incluimos a continuación, permiten incorporar el aprendizaje de competencias dentro de las asignaturas del curriculo. Como se deduce de la última de las cuestiones de reflexión de la tabla 4 , no es la única vía para que el estudiante adquiera o refuerce las competencias genéricas, pero la preocupación por incorporar estas cuestiones en los diseños curriculares es imprescindible, ya que facilita el proceso de aprendizaje integral. Ello no excluye, sino que complementa, otras prácticas habituales como la asistencia de los estudiantes a los cursos impartidos por diversas instancias universitarias (Institutos de Ciencias de la Educación u otros). ${ }^{8}$ El aprendizaje de una determinada competencia puede integrarse bien en la asignatura de

8 Sirvan de ejemplo los cursos que el Instituto de Ciencias de la Educación de la Universidad de Zaragoza ofrece a los estudiantes sobre temas como los siguientes: Técnicas de expresión oral, Técnicas para optimizar los recursos personales, materiales y temporales, Guía para crear la propia empresa, cómo superar un proceso de selección de personal, u otros. Igualmente merece destacarse la reciente iniciativa de algunas universidades, Carlos III y Universidad de Alcalá, de establecer programas de formación específicas sobre competencias genéricas para sus estudiantes. En el caso de la de Alcalá, está previsto que la enseñanza de las competencias sea estructurada y que los estudiantes que sigan los cursos correspondientes obtengan a su término un certificado que tendrá carácter de título propio. Son procedimientos que siguen la pauta establecida en el mundo universitario anglosajón, pionero en la incorporación explícita de las competencias genéricas a los aprendizajes universitarios. 
Geografía, ya que los diferentes contextos académicos -clases magistrales, seminarios, prácticas de campo o de laboratorio, o la realización de proyectos- se prestan a la realización de actividades que favorecen que la competencia seleccionada se enseñe, se integre en los demás contenidos, se practique, sea objeto de reflexión y, por fin, evaluada. No obstante, es complejo para el profesor, trasladar al aula las consideraciones que hemos realizado sobre el aprendizaje de competencias genéricas y diseñar la metodología adecuada para su enseñanza, el aprendizaje de los estudiantes y la evaluación del mismo (Lasnier, 2002).

En los departamentos de Geografía de las universidades británicas ya hace tiempo que se ha introducido plenamente el aprendizaje de competencias en los contenidos de las asignaturas cursadas por los futuros graduados. Algunos departamentos se han asociado formando la Geography Discipline Network. Resultado de su trabajo conjunto en esta línea es una amplia colección de publicaciones (Bradford, 2000; Burkill, et al; Gardiner y Hugues, 2000; Livingstone y Matthews, 2000), que constituyen aportaciones de gran interés. En ellas se confirma una vez más que la enseñanza de las competencias genéricas no es algo nuevo en el conjunto de las preocupaciones docentes de muchos profesores, y en concreto de los de Geografía. Eso sí, era necesario extraer estos contenidos del "currículo oculto" y hacer explícito su aprendizaje (Gravestock y Healey, 2000; Rodríguez Espinar, 2000).

No podemos dejar de advertir que la incorporación de competencias a las dife rentes asignaturas supondrá diseñarlas de nuevo, temporalizarlas, secuenciar las actividades y evitar que supongan una carga excesiva para los estudiantes. Como veremos más adelante, es necesario ser realistas al llevar a cabo las programaciones (Spronken-Smith, 2005), partiendo de un adecuado diagnóstico de la situación de los estudiantes en relación con la competencia genérica cuyo aprendizaje queremos incorporar al programa.

\subsection{El diagnóstico previo y su interés}

No plantea ninguna duda que un diseño adecuado de actividades encaminadas al aprendizaje de competencias debe ir precedido del diagnóstico previo y certero de cuáles son las carencias principales de los estudiantes, ya que permitirá conocer sus principales necesidades formativas. Hay muchas formas de realizar este diagnóstico. Una es la realización de una encuesta al iniciar el curso académico entre los matri-

Tabla 5. Etapas del diseño de las actividades para el aprendizaje de competencias genéricas.

\begin{tabular}{|c|c|c|c|c|}
\hline \multirow{2}{*}{$\begin{array}{l}\text { Competencia } \\
\text { seleccionada }\end{array}$} & \multirow{2}{*}{$\begin{array}{l}\text { Resultados del } \\
\text { aprendizaje }\end{array}$} & \multicolumn{2}{|c|}{ Evaluación } & \multirow{2}{*}{$\begin{array}{c}\text { Actividades para } \\
\text { la formación }\end{array}$} \\
\hline & & Evidencias & Criterios & \\
\hline
\end{tabular}


culados en la asignatura o en un conjunto de las mismas. Su estructura y complejidad depende de los recursos de los que el profesor disponga. La tarea se facilita mucho, si participan varios profesores o incluso toda un área o Departamento. Aunque es un procedimiento costoso, las ventajas de la encuesta como método de diagnóstico son claras: proporciona la posibilidad de realizar una cuantificación más exacta de las necesidades y carencias de los estudiantes y, por otra parte ayuda a estimular a los estudiantes y a que se involucren más en su aprendizaje personal. ${ }^{9}$

\subsection{Etapas en la preparación de actividades para el aprendizaje de competencias en las asignaturas del Grado en Geografía}

En la preparación de las actividades para el aprendizaje de competencias pueden seguirse distintas etapas interrelacionadas entre sí: identificación y secuenciación de

Tabla 6a. Resultados del aprendizaje en el nivel 1 (básico). Elaboración propia.

\begin{tabular}{|ll|}
\hline Competencia seleccionada & \multicolumn{1}{c|}{ Ejemplos de resultados de aprendizaje } \\
\hline Lectura comprensiva & Es capaz de identificar las ideas principales de un texto \\
& y de localizar un contenido específico. \\
& Identifica las principales cuestiones de una \\
& explicación. \\
& Es capaz de tomar notas mientras escucha. \\
& Es capaz de resumir los principales aspectos de una \\
& intervención. \\
& Es capaz de escribir de modo correcto en su idioma sin \\
Comunicación escrita & faltas de ortografía ni de expresión. \\
& Puede presentar una reseña escrita de forma clara, \\
& ordenada y concreta sobre un documento relacionado \\
& con la asignatura. \\
& Se expresa de manera clara y correcta. \\
Comunicación oral & Es capaz de exponer con claridad cuestiones \\
& relacionadas con la asignatura. \\
& Capta a su auditorio. \\
& Es capaz de trabajar con compañeros a los que conoce \\
Trabajo en equipo & y con los que tiene relación previa. \\
& Realiza las tareas que le son asignadas dentro del \\
& equipo \\
& Transmite a los demás eficazmente los resultados de la \\
& tarea asignada.
\end{tabular}

9 Como ya se ha dicho, las autoras de este artículos y su equipo distribuyeron una encuesta al total de los setenta y tres estudiantes de sus respectivas asignaturas. El diseño de la encuesta y sus principales conclusiones ya han sido objeto de diferentes publicaciones (GIDOCUZ, 2003 y 2004; Escalona y Loscertales, 2005). 
Tabla 6b. Resultados del aprendizaje en el nivel 2 (avanzado). Elaboración propia.

\begin{tabular}{|c|c|}
\hline Competencia seleccionada & Ejemplos de resultados de aprendizaje \\
\hline Lectura comprensiva & $\begin{array}{l}\text { Selecciona los textos adecuados para el fin propuesto. } \\
\text { Lee con profundidad y sentido crítico textos complejos. } \\
\text { Establece relaciones entre contenidos. }\end{array}$ \\
\hline Escucha activa & $\begin{array}{l}\text { Reflexiona y analiza mientras otros hablan. } \\
\text { Responde adecuadamente en diversos contextos. } \\
\text { Contribuye correctamente y en el momento oportuno en un } \\
\text { debate }\end{array}$ \\
\hline Comunicación escrita & $\begin{array}{l}\text { Elabora documentos complejos de forma sistemática y } \\
\text { rigurosa } \\
\text { Utiliza la terminologia propia de la disciplina } \\
\text { Dispone de recursos para preparar informes escritos en un } \\
\text { contexto profesional }\end{array}$ \\
\hline Comunicación oral & $\begin{array}{l}\text { Expone con claridad, autonomía y competencia profesional } \\
\text { un tema complejo. } \\
\text { Conoce y es capaz de utilizar los recursos de un buen } \\
\text { orador. } \\
\text { Se apoya de forma adecuada en los medios audiovisuales } \\
\text { oportunos. }\end{array}$ \\
\hline Trabajo en equipo & $\begin{array}{l}\text { Es capaz de ejercer el liderazgo o cualquier otra función de } \\
\text { responsabilidad dentro del equipo. } \\
\text { Toma iniciativas respondiendo a otras aportaciones de sus } \\
\text { compañeros o del profesor en tutoría. } \\
\text { Asume el modo y la dinámica de trabajar en equipo y adopta } \\
\text { un comportamiento serio y profesional. }\end{array}$ \\
\hline
\end{tabular}

los resultados del aprendizaje, determinación de las pruebas o evidencias que se pedirán a los estudiantes para acreditar su aprovechamiento así como de los procedimientos y criterios con los que van a ser evaluadas; y diseño de las actividades mediante las cuales va a facilitarse la consecución de los resultados previstos y la superación de la correspondiente evaluación (Cruz, 2004; Moon, 2004). Es importante una adecuada y coherente relación entre los objetivos que se pretenden alcanzar, las actividades académicas diseñadas para lograrlos, y las evidencias y procedimientos elegidos para evaluar si el estudiante ha obtenido o no los resultados esperados (tabla 5).

A continuación aportamos diversos ejemplos de estas etapas en relación con el aprendizaje de las competencias genéricas: trabajo en equipo, lectura comprensiva, comunicación escrita, escucha activa y comunicación oral. Como ya se ha expuesto (v. tabla 3) el trabajo en equipo se considera como una competencia con un déficit formativo medio, por lo que parece oportuno reforzar su aprendizaje y además incor- 
porar al mismo aquellas capacidades que son necesarias para cooperar con especialistas de otras disciplinas, ya que en esta última competencia el déficit formativo es muy alto. La comunicación escrita y oral en la propia lengua, también es una de las competencias genéricas más valoradas. Como muchas otras, es una competencia

Tabla 7a. Relación de evidencias para la evaluación de los aprendizajes (nivel 1 o básico). Elaboración propia.

\begin{tabular}{|c|c|}
\hline Competencia seleccionada & Evidencias del aprendizaje \\
\hline Lectura comprensiva & Incorporadas en las restantes evidencias. \\
\hline Comunicación escrita & $\begin{array}{l}\text { Reseñas críticas sobre artículos de prensa de actualidad } \\
\text { que tengan relación con la asignatura. } \\
\text { Resumen de un artículo científico o capítulo de libro. } \\
\text { Ensayo a partir de la síntesis de textos diversos. } \\
\text { Examen escrito sobre los contenidos de la materia } \\
\text { correspondiente. }\end{array}$ \\
\hline Escucha activa & $\begin{array}{l}\text { Anotaciones realizadas sobre una exposición, } \\
\text { conferencia o proyección relacionada con la materia. }\end{array}$ \\
\hline Comunicación oral & $\begin{array}{l}\text { Exposición en clase a sus compañeros de una cuestión } \\
\text { previamente estudiada. }\end{array}$ \\
\hline Trabajo en equipo & $\begin{array}{l}\text { Realización de un trabajo de carácter bibliográfico } \\
\text { sobre una cuestión del programa. } \\
\text { Realización de una breve memoria descriptiva acerca } \\
\text { del modo en que el equipo ha trabajado. }\end{array}$ \\
\hline
\end{tabular}

Tabla 7b. Relación de evidencias para la evaluación de los aprendizajes (nivel 2 o avanzado). Elaboración propia.

\begin{tabular}{|ll|}
\hline Competencia seleccionada & \multicolumn{1}{c|}{ Evidencias del aprendizaje } \\
\hline Lectura comprensiva & Incorporadas en las restantes evidencias. \\
Comunicación escrita & Recensión crítica de artículos científicos. \\
& Ensayo de carácter bibliográfico complejo. \\
& Informe sobre un trabajo de carácter empírico. \\
& Memoria de un proyecto de investigación. \\
Escucha activa & Síntesis crítica sobre una conferencia fuera del aula. \\
Comunicación oral & Earticipación en un debate. \\
& Exposición más extensa que la del nivel 1 sobre un tema de \\
Trabajo en equipo & la asignatura. \\
& Realización de un trabajo de carácter bibliográfico o empí- \\
& rico sobre una cuestión más compleja que en el nivel ante- \\
& rior. \\
& Realización de una memoria descriptiva completa y razonada \\
& acerca del modo en que el equipo ha trabajado. \\
\hline
\end{tabular}


compleja, que integra diversas capacidades y habilidades. Efectivamente, ser buenos comunicadores significa también ser buenos lectores y buenos escuchadores. Por ello, seguimos la pauta establecida en otras universidades (Burkill, et al. 2000), y proponemos una metodología para trabajar específicamente la capacidad para leer comprensivamente y escuchar de forma activa.

Por otra parte, hemos seleccionado la lectura comprensiva como competencia por la percepción de las fuertes carencias que tienen los estudiantes. Sin mejorarla, difícilmente podrán adquirir esos conocimientos tan necesarios para su futura cualificación profesional, ni tampoco adquirirán otras competencias básicas íntimamente relacionadas con ésta, como la capacidad de análisis y de síntesis. Es, por lo tanto, junto con la escucha activa, un pilar básico tanto para el conjunto de las competencias de comunicación como para el posterior refuerzo de otras competencias.

Estas competencias han sido también seleccionadas porque pueden ser practicadas en cualquier asignatura del título de grado en Geografía y permiten utilizar para ello, las actividades académicas más habituales, como las exposiciones teóricas a cargo del profesor, los seminarios, las prácticas de campo y de laboratorio, o la realización de proyectos (Moreno y Marrón, 1995).

\subsubsection{La identificación de los resultados del aprendizaje ${ }^{10}$}

El diseño de la enseñanza de una competencia genérica debe apoyarse en la espe cificación por el profesor de los resultados que espera que alcancen sus estudiantes. ${ }^{11}$ Éstos han sido estructurados en dos niveles: nivel 1 (básico) y nivel 2 (avanzado), buscando un adecuado equilibrio entre el curso en que se encuentra el estudiante y el nivel de exigencia. Las tablas 6 a y $6 \mathrm{~b}$ incluyen el enunciado de los resultados que el estudiante debe alcanzar en cada una de las capacidades y niveles. Se asume que los estudiantes del nivel 2 han alcanzado ya los resultados de aprendizaje propios del nivel 1 .

10 El concepto de resultados de aprendizaje así coo su adecuada utilización es uno de los aspectos que más atención está recibiendo en el proceso de divulación de las nuevas pautas docentes en el marco del Espacio Europeo de Educación Superior. En la página web oficial de dicho proceso, www hologna-bergen2005.no, pueden encontrarse documentos de gran interés -a los que renitimos- sobre el estado de esta cuestión en la universidades europeas, resultantes de diversos seminarios específicos, como el celebracto en Edimburgo en julio de 2004 ("Using learning outcomes").

11 Estos resultados pueden ser perfectamente asumidos por profesores que enseñen estas competencias genéricas en asignaturas de titulaciones afines. 


\subsubsection{La selección de las evidencias de aprendizaje}

Como expresa el proceso expuesto en el esquema de la tabla 5, la consecución de los resultados de aprendizaje se aprecia a partir de diversas pruebas o evidencias y de su evaluación mediante criterios previamente establecidos y conocidos por los estudiantes. Lógicamente éstos deberán ser informados al comienzo del curso de las diferentes tareas que tendrán que presentar. A su vez, el conocimiento de los procedimientos y criterios con los que van a ser evaluados, les servirá de orientación para enfocar su aprendizaje y aprovechar al máximo las actividades académicas que realicen. Proponemos diversos ejemplos de cuáles podrían ser algunas de las pruebas o evidencias según la competencia que se haya incorporado al aprendizaje y el nivel básico (1) o avanzado (2) del estudiante (tablas 7a y 7b). No se aportan ejemplos de evidencias propias de la capacidad de lectura comprensiva, ya que ésta capacidad se incluye siempre en la tarea de la preparación de cualquier evidencia de otra competencia: un documento escrito, la participación en un debate, una exposición oral a partir de una documentación previa, entre otras.

Este tipo de pruebas son habituales en la práctica universitaria; su objetivo es evaluar el aprendizaje de los contenidos propios de una asignatura, es decir, de las competencias específicas que en ella se adquieren o refuerzan. Pero, como se deduce de su incorporación a las tablas $7 \mathrm{a}$ y $7 \mathrm{~b}$, también sirven para apreciar si el estudiante ha alcanzado los resultados de aprendizaje establecidos para la competencia genérica correspondiente. Por ejemplo, un trabajo de carácter bibliográfico o empírico sobre una cuestión compleja, a la vez que aporta una evidencia adecuada del aprendizaje de contenidos de una asignatura, es también evidencia del aprendizaje de la competencia comunicación escrita o, si se expone de forma oral, de la competencia comunicación oral. Por ello para su evaluación deberán establecerse criterios adecuados que permitan apreciar si el estudiante ha alcanzado los resultados de aprendizaje previstos en ambos casos. La elaboración de unas plantillas que faciliten una evaluación correcta, objetiva y homogénea puede ser de gran utilidad tanto para el profesor como para los estudiantes. A modo de ejemplo se incorpora una tabla de criterios para evaluar en un trabajo de carácter bibliográfico complejo los resultados del aprendizaje de competencias específicas asî como los de las competencias genéricas lectura comprensiva y comunicación escrita (v. tabla 8).

Este tipo de plantilla servirá, adaptando los contenidos, para evaluar las demás pruebas o evidencias de aprendizaje propuestas. 
Tabla 8. Criterios para la evaluación del nivel de competencias genéricas y específicas alcanzado en un trabajo de carácter bibliográfico.

Elaboración propia.

\begin{tabular}{|c|c|c|c|c|c|c|}
\hline $\begin{array}{l}\text { Criterios para evaluar } \\
\text { competencias especificas }\end{array}$ & 1 & 0,75 & 0,5 & 0,25 & 0 & \\
\hline $\begin{array}{l}\text { Criterio 1: se cumple } \\
\text { Criterio 2: se cumple } \\
\text { Criterio 3: se cumple } \\
\text { Criterio 4: se cumple } \\
\text { Criterio 5: se cumple } \\
\text { Criterio 6: se cumple } \\
\text { Criterios para evaluar la capa- } \\
\text { cidad de lectura comprensiva } \\
\text { Asimilación de textos y otros } \\
\text { materiales consultados } \\
\text { Análisis crítico } \\
\text { Criterios para evaluar la calidad } \\
\text { de la comunicación escrita } \\
\text { La argumentación es sistemática } \\
\text { La argumentación es rigurosa }\end{array}$ & & & & & & $\begin{array}{l}\text { No se han asimilado } \\
\text { No se han analizado } \\
\text { Es desordenada } \\
\text { No lo es }\end{array}$ \\
\hline
\end{tabular}

\subsubsection{La preparación de actividades para el aprendizaje de competencias genéricas}

Éstas deben ser planteadas para proporcionar a los estudiantes los recursos adecuados que les faciliten superar con éxito las pruebas según los umbrales, al menos mínimos, establecidos en la evaluación (Moon, op.cit.: 3). La tabla 9 presenta una relación de algunas de las actividades que pueden ser realizadas para favorecer la práctica de las diferentes capacidades relacionadas con el trabajo en equipo y la comunicación. Por ello se ha procurado que fueran variadas. En todo caw), el profesor podrá seleccionar las que le parezcan más adecuadas. Los niveles básico y avanzado los marcará siempre el profesor mediante un diferente grado de complejidad y de exigencia.

Al seleccionar las actividades de aprendizaje hay que tener en cuenta que se les deberá asignar un tiempo suficiente para que puedan ser explicadas a los estudiantes adecuadamente y para que éstos puedan practicarlas; el objetivo final será siempre conseguir el afianzamiento o la mejora de cada una de las competencias seleccionadas. Pongamos como ejemplo la realización en clase de una actividad para el aprendizaje y mejora de la capacidad de lectura comprensiva con estudiantes de nivel inicial. En relación con esta actividad se puede pedir también al estudiante que lleve a cabo una selección de fuentes para realizar un pequeño trabajo. En estos casos suele 
Tabla 9. Actividades para el aprendizaje de competencias genéricas. Elaboración propia.

\begin{tabular}{|c|c|}
\hline \begin{tabular}{|l|} 
Competencia \\
seleccionada
\end{tabular} & Actividades para el aprendizaje \\
\hline Lectura comprensiva & $\begin{array}{l}\text { Presentación de técnicas de lectura } \\
\text { Sesiones de lectura en clase de textos sobre la signatura aplicando } \\
\text { las distintas técnicas explicadas. } \\
\text { Elaboración de resúmenes escritos u orales como comprobación. }\end{array}$ \\
\hline Comunicación escrita & $\begin{array}{l}\text { Distribución de una ficha con pautas para la correcta elaboración de } \\
\text { un documento escrito. } \\
\text { Examen, a modo de ejemplo, de diferentes documentos escritos } \\
\text { relacionados con la asignatura. } \\
\text { Práctica del modo de preparar los diferentes tipos de documentos } \\
\text { escritos }\end{array}$ \\
\hline Escucha activa & $\begin{array}{l}\text { Distribución de una ficha con pautas para conseguir una escucha } \\
\text { activa. } \\
\text { Formulación de preguntas a los estudiantes en el transcurso de una } \\
\text { exposición teórica del profesor. } \\
\text { Asistencia a conferencias relacionadas con la materia. } \\
\text { Proyección de documentales en clase. } \\
\text { Sesiones de debate. }\end{array}$ \\
\hline Comunicación oral & $\begin{array}{l}\text { Distribución de una ficha con pautas para la correcta realización de } \\
\text { una exposición oral. } \\
\text { Taller preparatorio a cargo de un especialista en cuestiones de } \\
\text { comunicación. }\end{array}$ \\
\hline Trabajo en equipo & $\begin{array}{l}\text { Debate entre los estudiantes sobre su experiencia al trabajar en } \\
\text { equipo con otros compañeros. } \\
\text { Distribución de una guía sobre el trabajo en equipo } \\
\text { Presentación y explicación de la misma en clase por el profesor } \\
\text { Sesiones de trabajo del grupo supervisadas por el profesor, en las } \\
\text { que se prestará especial atención a algún aspecto del trabajo en } \\
\text { equipo en que se quiera insistir (manejo del tiempo, eficacia de las } \\
\text { reuniones, entre otras) } \\
\text { Presentación al profesor de breves informes periódicos sobre el fun- } \\
\text { cionamiento del equipo o sobre los resultados obtenidos. }\end{array}$ \\
\hline
\end{tabular}

ser necesario consultar sumarios de revistas o de los contenidos de un libro recapitulativo. Para ello es útil aplicar una técnica de lectura rápida como scanning (identificación del contenido específico). ${ }^{12}$ Para practicar esta competencia el profesor puede llevar a cabo una actividad siguiendo las pautas que se indican en la tabla 10.

12 Basado en Burkill, S., Corey, D. y Healey, M. (2000): Improving student communications skills. Cheltenham, Geography Discipline Network, Cheltenahm and Gloucester College of Higher Education, pág. 47 y ss. 
Tabla 10. Pautas para la realización de una actividad de aprendizaje.

Actividad para el aprendizaje de la Lectura comprensiva: Práctica del scanning
1. Dar a cada uno de los estudiantes una ficha explicativa de la técnica. ${ }^{13}$
2. Explicación por el profesor de su finalidad y aspectos básicos.
3. Distribución individual o por grupos de materiales bibliográficos para su examen.
4. Mostrar y enseñar el funcionamiento de la biblioteca del Departamento o del Centro.
5. Aplicación de la técnica por los estudiantes para localizar en un texto o monografía la
información requerida.
6. Discusión en pequeños grupos de los resultados.
7. Puesta en común en clase.

\section{Conclusión}

Trabajando de la manera que se ha venido exponiendo se cubren diferentes objetivos; en primer lugar se adopta la metodología recomendada para conseguir la convergencia universitaria europea y el establecimiento efectivo del Espacio Europeo de Educación Superior. El aprendizaje de competencias específicas y genéricas va a ser un referente común para la formación de los estudiantes de las diferentes titulaciones y sistemas universitarios europeos. Cada vez más, enseñar debe consistir no sólo en ir transmitiendo conocimientos teórico - prácticos cada vez más complejos, sino en formar en el más amplio sentido del término a los ciudadanos del futuro.

En segundo lugar se han dado diversas pautas para incorporar la enseñanza de las competencias genéricas en el aprendizaje de las asignaturas del grado de Geografía. No hay duda de que así no sólo se mejora el proceso de enseñanza-aprendizaje, sino también la profesionalidad y capacidad de hacer bien las cosas por los titulados y, en consecuencia, su futura inserción laboral. Las diferentes tablas y plantillas intercaladas en el texto, pretenden ayudar a comprender esta metodología, y proporcionar a los profesores interesados unos materiales que puedan adaptar a sus necesidades.

Queremos destacar que trabajar de este modo no es fácil, que existen múltiples inconvenientes reales para trasladar a las aulas lo que en principio podrían considerarse meras quimeras, reflexiones más o menos utópicas. Según nuestra experiencia personal en este campo, el resultado final ha sido siempre, sin embargo, gratificante, aunque lógicamente los logros de los estudiantes no hayan sido homogéneos. No podemos dejar de mencionar que en este proceso son esenciales los diferentes aspec-

13 Esta técnica de lectura está descrita en Burkill, et al (2000) así como en otras numerosas publicaciones que el profesor interesado encontrará fácilmente. 
tos interrelacionados reflejados en la tabla 5, es decir, el nivel del estudiante, los resultados de aprendizaje establecidos según dicho nivel, la forma como serán evaluados y las actividades académicas previstas para facilitar su consecución. De su correcta interrelación dependerá en gran parte el éxito final.

A las autoridades académicas universitarias responsables del diseño de la titulación corresponderá dar previamente las pautas oportunas sobre la estructura de las enseñanzas; es decir, sobre su organización en cursos, módulos y créditos ( $v$. Tabla 4). Valga como conclusión final la afirmación de que todos, en la Universidad, nos vamos a ver involucrados en el rediseño de nuestras asignaturas y que es conveniente estar preparados para ello.

\section{Bibliografía}

Agencia Nacional de Evaluación de la Calidad y de Acreditación (2004): Título de grado en Geografia y Ordenación del Territorio. Madrid.

Barton, L. (1996): La taxonomía de Bloom y el pensamiento crítico. http://www.eduteka.org (consulta: 15/7/2005).

Bradford, M. (2000): Improving students' team and personal skills. Gloucester, Geography Discipline Network, Geography \& Environmental Research Unit, and Cheltenham \& Gloucester College of Higher Education.

Burkill, S., Corey, D. y Healey, M. (2000): Improving students' communications skills. Gloucester, Geography Discipline Network, Geography \& Environmental Research Unit, and Cheltenham \& Gloucester College of Higher Education.

Cruz Tomé, M.A. de la (2004): El proceso de aprendizaje-enseñanza de competencias. Zaragoza, Universidad de Zaragoza, Instituto de Ciencias de la Educación.

Cruz Tomé, M.A. de la (2005): Taller sobre el proceso de aprendizaje-enseñanza de competencias. Zaragoza, Universidad de Zaragoza, Instituto de Ciencias de la Educación (mecanografiado).
Escalona Orcao, A.I. y Loscertales Palomar, B. (2005): Actividades para la enseñanza y el aprendizaje de competecias en el marco del Espacio Europeo de Educación Superior. Zaragoza, Prensas Uiversitarias de Zaragoza, col. Textos docentes, $\mathrm{n}^{\circ} 110$.

Fallows, S. y Steven, C. (2000): Integrating key skills in Higuer education; employability, transferable skills and learning for life. Londres, Kogan Page.

Fundación Universidad-Empresa (1999): Guía de las empresas que ofrecen empleo. Madrid, Fundación Universidad-Empresa.

Fundación BBVA (2004): Segundo estudio de la Fundación BBVA sobre los universitarios españoles. Madrid, Fundación BBVA.

García Valcárcel, A., coord. (2001): Didáctica Universitaria. Madrid, Narcea.

Gardiner, V. y Hughes, K. (2000): Improving students problem-solving and thinking skills. Gloucester, Geography Discipline Network, Geography \& Environmental Research Unit, and Cheltenham \& Gloucester College of Higher Education.

GIDOCUZ (Escalona, A; Loscertales, B; Lardiés, R. y Lacosta, A.) (2003): "Aportación metodológica para favorecer el 
aprendizaje de competencias genéricas en la licenciatura de Geografía". En Marrón Gaite, Ma.J., Moraleda Nieto, C. y Rodríguez de Gracia, H. (eds.): La enseñanza de la Geografia ante las nuevas demandas sociales. Toledo, Grupo de Didáctica de la Asociación de Geógrafos Españoles, Universidad de Castilla-La Mancha y Escuela Universitaria de Magisterio de Toledo, 419-432.

GIDOCUZ (Escalona, A; Loscertales, B; Lardiés, R. y Lacosta, A.) (2004): Aprendizaje de competencias clave mediante trabajo en equipo. Una experiencia en el Departamento de Geografía de la Universidad de Zaragoza. En Libro de Resü. menes. III congreso Internacional Docencia Universitaria e Innovación. Gerona, Universitat de Girona, volumen segundo, 212.

Gravestock, P. y Healey, M. (2000): "Editors preface". En Burkill, S., Corey, D y Healey, M.: Improving students' communication skills. Gloucester, Geography Discipline Network, Geography \& Environmental Research Unit, and Cheltenham \& Gloucester College of Higher Education, ix-xii.

Lasnier, F. (2000): Réussir la formation per competences. Montréal, Guerin.

Livingstone, I. y Matthews, H. (2000): Assessing and recording a skills-based curriculum. Gloucester, Geography Discipline Network, Geography \& Environmental Research Unit, and Cheltenham \& Gloucester College of Higher Education.

Monereo, C. y Pozo, J. I., eds. (2003): La Universidad ante la nueva cultura educativa. Enseñar y aprender para la autonomía. Madrid, Síntesis
Moon, L. (2004): Linking levels, Learning Outcomes and Assesment Criteria. Edimburgo, Seminario Tuning sobre resultados de aprendizaje.

Moreno Jiménez, A. y Marrón Gaite, M.J. (1995): Enseñar Geografia. De la teoria a la prâctica. Madrid, Síntesis.

Rodríguez Espinar, S. (2000): El diseño de proyectos de innovación docente. Zaragoza, Instituto de Ciencias de la Educación.

Spronken-Smith, R. (2005): Implementing a problem.based learning approach for teaching research methods in geography. Journal of Geography in Higuer Education, 29 (2): 203-221.

Tuning Management Committee (2004): Student workload, teaching methods and learning outcomes: the tuning approach. En Proyecto Tuning, fase 2 WwW.relint.deusto.es/TUNINGProject/ind ex_english.htm (consulta: 20/7/2005s).

Wagenaar, R. (2003): The tuning methodology. Learning outcomes and competences. (www.relint.deusto.es/TUNINGProject/pr esentations/presentations_phase 2/PRESENTACION3.pdf)

VV.AA. (2003): Tuning educational structures in Europe. Informe Final, Proyecto Piloto fase 1 (www.relint.deusto.es).

V.AA. (2005): La universidad y el Espacio Europeo de Educación Superior (número monográfico), Revista española de Pedagogía, no 230 .

Zabalza Beraza. M.A. (2003): Competencias docentes del profesorado universitario. Madrid, Narcea. 